\title{
A Stable TORA Based for Routing in Mobile Ad Hoc Networks
}

\author{
Sajjad Jahanbakhsh Gudakahriz \\ Department of Computer \\ Engineering \\ Germi Branch \\ Islamic Azad University \\ Germi, Iran \\ Sa_jahanbakhsh@iaugermi.ac.ir
}

\author{
Shahram Jamali \\ Computer Engineering \\ Department \\ University of \\ Mohaghegh Ardabili \\ Ardabil, Iran \\ Jamali@iust.ac.ir
}

\author{
Mina Vajed Khiavi \\ Department of Computer \\ Engineering \\ Germi Branch \\ Islamic Azad University \\ Germi, Iran \\ M_vajed@yahoo.com
}

\author{
Alireza Soleimani \\ Department of Computer \\ Engineering \\ Meshkin Shahr Branch \\ Islamic Azad University \\ Meshkin Shahr, Iran \\ Alireza.soleimany@yahoo.com
}

\begin{abstract}
A mobile ad hoc network (MANET) is defined as a set of mobile nodes which communicate through wireless links with no central infrastructure. In these networks nodes are always moving and could enter to or exit from the network at any time. Therefore, network topology is completely dynamic. Another characteristic of these networks is the low node energy level, resulting from inherent node limitations. Stable routing is one method to face routing challenges in mobile ad hoc networks. The objective of this study is to stabilize TORA protocol which is a distributed routing protocol, with high adaptation, efficiency, and suitability for large and dense mobile ad hoc networks and thus to provide a new high efficiency protocol. The proposed protocol introduced as Stable-TORA has been compared with base protocol TORA in different scenarios via NS-2 simulator environment and its efficiency is evaluated.
\end{abstract}

Keywords-mobile ad hoc networks; routing; stable routing; TORA protocol

\section{INTRODUCTION}

Ad hoc networks are possible to implement when connecting one or more computers equipped with wireless network cards [1, 2]. A mobile ad hoc network (MANET) can configure its self on the fly. Infrastructure access points are not used in the wireless ad hoc topology in which, each computer can act either as a node or as a wireless access point / routing agent. Ad hoc networks are not usually used for long-term applications because they have relatively low stability and that is why they are used temporarily. Routing in MANETs is always considered as a challenging issue. In MANETs, nodes have not previous knowledge of network topology. Therefore, they have to discover the destination's location in order to communicate with other nodes. The general idea is that a new node optionally broadcasts its presence to the network and listens to its neighbors. So the node partially gets informed about its close nodes and as this happens throughout the network, a node ends up learning all other nodes or at least knows a way to reach them [3-5]. One group of MANETs routing protocols is the table driven protocols. In this routing method, each node acquires routing information via saving other nodes' local information and this information is used to data transfer through different nodes [6]. Another group of MANETs routing protocols is the on demand protocols. In this group, the route between two nodes is formed when there is a necessity for communication between two nodes and they are short lived. When there is need for a route by a source node, if it does not have a valid route for the destination node, it will start the process of discovering the path to reach the destination. It is possible for a mid-node to have a route to the destination [6].

Due to the dynamic topology of MANETs, discovered routes may vanish anytime and then there would be a need for re-routing. Quick changes in topology would lead to increased need of re-routing and this would decrease the network efficiency. One of the methods to overcome this problem is stable routing. The objective of stable routing is that during route discovery the established routes would have high stability with longer durability. This will cause reduction in need for rerouting and increase the network efficiency [7, 8]. Stable routing protocols provided for MANETs are a rather recent research field. According to recent analytic results, lifetime of a route, reflecting the route stability depends on route length and lifetime of each link in the route. In [9-12] a few stable routing protocols are presented. The research objective in the current paper is to provide a stable routing protocol for ad hoc networks.

\section{TORA AND STABLE-TORA}

TORA protocol [13] is a distributed routing protocol for mobile ad hoc networks. This protocol has high adaptation, is efficient and scalable. TORA is run in three major phases: route forming, route maintaining and route deleting. Forming a route from one node to another needs publishing some directed link from the source node to the destination node. This is only done when a node with direction-less links needs a route to destination. Therefore route forming is a process of giving direction to links in a partially or fully direction-less network. 
The method used for this goal is done via the query/reply process defined in [14], which makes a directed acyclic graph (DAG) with root at destination. Such a DAG is known as a destination rooted DAG. Route maintenance is the reaction against topology changes in the network, in a way that in limited time routes should be established again. TORA is efficient in responding to topology changes and is able to recognize network splitting. This leads to the third phase which is route deleting. During the recognition of network splitting, in order to clear invalid routes, their links should change to nondirected links. Protocol run these three phases using three distinct control packets including: query packet (QRY), update packet (UPD), and clear packet (CLR).

Routing in mobile ad hoc networks suffers major challenges including node mobility, dynamic topology, and limited node battery capacity. Mobility of the nodes causes rapid changes in network topology and designed routing algorithms should adapt the routing based on this dynamic topology. Limited node battery capacity is another challenge, based on which routing algorithms should send via different routes in a balanced manner, so the node batteries discharge with a similar rate. That way network lifetime increases. Increase in network lifetime would lead to increase in network efficiency [15]. One of the efficient methods to improve routing in mobile ad hoc networks is to select routes with high stability [16]. This would result in decrease in packet reduction, routing delay, and routing overhead, since the need for rerouting is reduced. In this part the objective is to improve TORA routing protocol via discovering and using stable routes. The introduced protocol is called Stable-TORA where route stability, in terms of route links lifetime and nodes energy level, is considered during route discovery. In fact the most important criteria to evaluate stability of a route are route links' lifetime and nodes' energy level [16]. Link lifetime parameter indicates how long the link between two nodes will be available and energy parameter also shows how long the node will be online. Therefore, we consider these two parameters as the main factors in route stability and by applying them the Stable-TORA protocol is produced.

\section{A. Stable-TORA Protocol}

TORA protocol is a multipath routing protocol for MANETs. This protocol discovers all routes available between the source node and the destination node, and among discovered routes it uses the shortest route to send and receive data. The problem of this protocol is that during discovery of all possible routes between the source and destination it is possible some routes to vanish in near future and the discovery of these routes leads only to overhead and efficiency reduction. In order to solve this problem, it is planned that during routing the discovered and established routes would be those that are high in terms of stability and have low probability of vanishing in the near future. In order to find routes with high stability in TORA protocol it is necessary to add these parameters to the record of each node:

- Parameter of minimum lifetime of a link between two nodes: this parameter would be added to the record of each node as min_ttl and shows the minimum lifetime of link in the route.

- Parameter of minimum energy of route nodes: would be added to the record of each node as min_energy and shows minimum energy of the nodes in the route.

- Parameter of route suitability would be added to the record of each node as RS. It will show the amount of suitability and quality of the route.

Routing procedure in Stable-TORA protocol would ignore repeated packets during sending route query packets to discover distinct node routes. Also each node calculates its remaining energy percentage and also the lifetime of the link between it and the previous node when it receives a route query packet. If remaining energy percentage of this node is less than minimum energy of route query packet, it will put this amount in min_energy field, otherwise it will put the amount of route query packet in min_energy field. It also compares link lifetime with amount in received route query packet and repeats the above mentioned task to initialize minimum lifetime of the link. When route query packet arrives to the destination neighborhood, the suitability of the route is calculated according to (1) and if the route has the desired suitability, the packet suitability will be added to the demand of route reply and a route reply packet will be sent towards source. If the route query packet has not the desired suitability, it will not be answered and the route will not be discovered.

$$
\mathrm{RS}=(\mathrm{E} \times \mathrm{T}) / \mathrm{H}
$$

where $\quad \mathrm{E}=$ Min_Energy, $\quad \mathrm{T}=$ Min_ttl/Maximum $\mathrm{ttl}$, $\mathrm{H}=$ Hopcount $/ \mathrm{N}$. In (1), energy and link stability are put in numerator since these parameters have direct relationship with route quality and suitability, while route length goes to denominator since route quality and suitability have converse relationship with route length. Also, min_energy is the percentage of remaining energy of each node, so it is a number between zero and one. On the other hand, in order to change the amount of link lifetime and route length to zero-one interval, we divide these two parameters to their maximum possible amount resulting for these parameters to have a similar to min_energy effect on calculating route suitability.

Designed Stable-TORA protocol includes these stages:

1) Sending route query packets from source to destination in distributed way.

2) Removing repeated route query packets in nodes.

3) Adding minimum energy of route nodes and minimum lifetime of route links to route query packet.

4) Wherever there is a possibility for sending route reply packet, route suitability is calculated via (1) and if RS is higher than a threshold, it means that this route has the desired suitability, a route reply packet is sent back to the source and route is discovered. If route suitability calculated via (1) is lower than the threshold, this route is considered not to have the desired suitability and no route reply packet is sent. 
5) After calculating the route suitability and in case of having one, RS value obtained by (1) is added to the route reply packet and is sent to the source. If there are different routes, the source uses the route with the highest suitability.

\section{EFFICIENCY EVALUATION}

In order to investigate the effects of the provided idea, we run some simulations in NS-2 simulator [17]. In order to implement Stable-TORA protocol, we applied the mentioned changes on TORA protocol. In order to evaluate, we simulated Stable-TORA protocol alongside with TORA protocol in terms of different scenarios and compared the efficiency of these two protocols based on these criteria:

- Packet delivery ratio: is the ratio of data packets delivered to destination to data packets sent from the source [18].

- End to end delay: this criterion equals to the mean time required for each data packet to travel from the source node to the destination node [18].

- Routing overhead: this equals to the total number of routing packets in the network [19].

- Network lifetime: the time the first node runs out of energy [20].

\section{A. First Simulation Scenario: Efficiency Criteria against the Number of Nodes}

In this scenario, the number of nodes has been considered variable and equal to $30,40,50$, and 60 . Simulation parameters have been presented in Table I and the simulation results are shown in Figures 1 to 4 . Based on the simulation results, we conclude that Stable-TORA protocol has better performance than TORA regarding the number of nodes. It has increased performance in the parameters of quality of packet delivery ratio and network lifetime, and has better end to end delay and routing overhead.

\section{B. Second Simulation Scenario: Efficiency Criteria Based on Different Pause Times}

In this scenario, the pause time has been considered variable. We simulated the produced different pause time scenario for 500 seconds with TORA and Stable-TORA protocols. Adjusted parameters have been presented in Table II and simulation results have been shown in Figures 5 to 8 .

TABLE I. FIRST SIMULATION SCENARIO PARAMETERS

\begin{tabular}{|c|c|}
\hline Parameters & Parameter Values \\
\hline Environment & $1000 \mathrm{~m} \times 1000 \mathrm{~m}$ \\
\hline Nodes number & $30,40,50,60$ \\
\hline Routing protocol & TORA and Stable-TORA \\
\hline Transmission range & $250 \mathrm{~m}$ \\
\hline Simulation time & 500 second \\
\hline MAC layer standard & 802.11 \\
\hline Traffic type & CBR (UDP) \\
\hline Buffer size & 50 Packets \\
\hline Nodes pause time & 0 Seconds \\
\hline Node moving speed & $20 \mathrm{~m} / \mathrm{s}$ \\
\hline Nodes position & Random \\
\hline
\end{tabular}

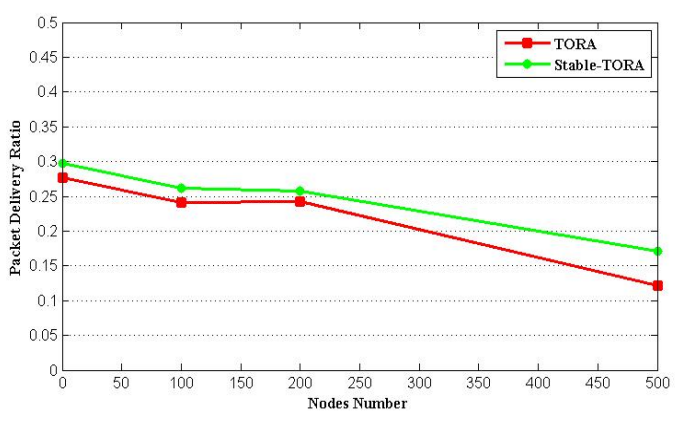

Fig. 1. Packet delivery ratio versus node number.

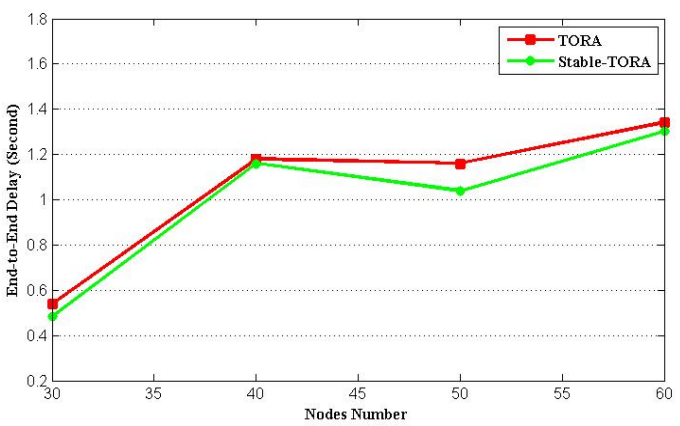

Fig. 2. End-to-end delay versus node number.

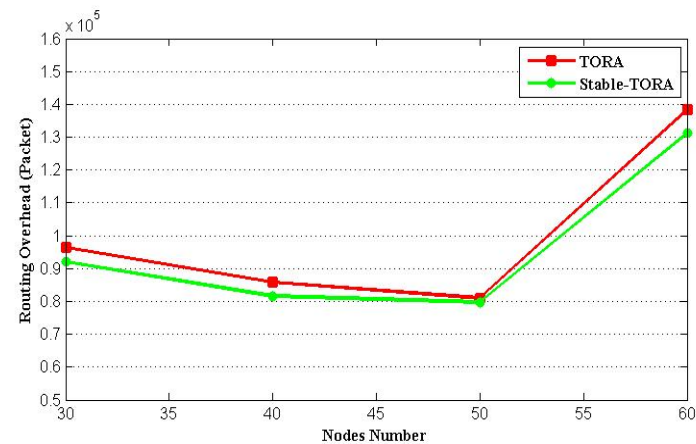

Fig. 3. Routing Overhead versus Nodes Number.

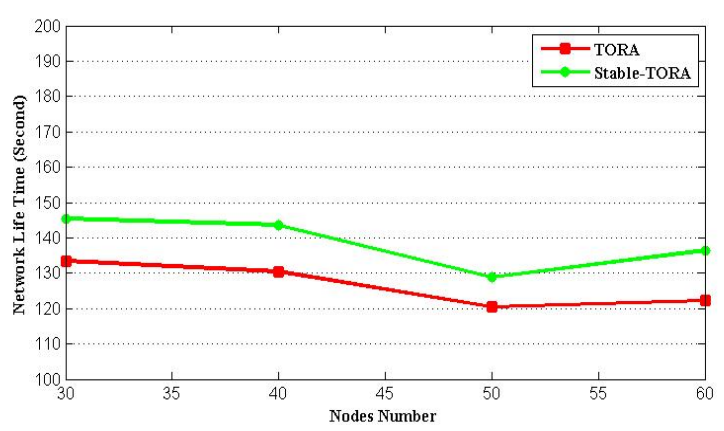

Fig. 4. Network life time versus node number.

Different pause times have been suggested as a researched parameter in order for the protocols to be evaluated in different statuses in terms of topological dynamicity. In Figures 5-8 we see that Stable-TORA has better packet delivery ratio, smaller 
end-to-end delay, smaller overhead and greater network life time. Based on these, we conclude that changes applied on TORA protocol in order to provide a new protocol, have caused better results and the new protocol shows suitable and better performance. We can summarize by saying that the Stable-TORA provided protocol, performs better in different situations compared with TORA in terms of packet delivery ratio, end to end delay, routing overhead and network lifetime. Better Stable-TORA performance is due to that the routes discovered, which have better quality in terms of route nodes energy, route links lifetime, and route length, are proved to be more efficient and to have higher stability and lifetime. Therefore packets are delivered through more reliable routes and have higher possibility of reaching destination. Also, the long route lifetime reduces the need for re-routing.

TABLE II. SECOND SIMULATION SCENARIO PARAMETERS

\begin{tabular}{|c|c|}
\hline Parameters & Parameters Values \\
\hline Environment & $1000 \mathrm{~m} \times 1000 \mathrm{~m}$ \\
\hline Nodes number & 50 \\
\hline Routing protocol & TORA and Stable-TORA \\
\hline Transmission range & $250 \mathrm{~m}$ \\
\hline Simulation time & 500 second \\
\hline MAC layer standard & 802.11 \\
\hline Traffic type & CBR $(\mathrm{UDP})$ \\
\hline Buffer size & 50 Packets \\
\hline Nodes pause time & $0,100,200,500$ seconds \\
\hline Node moving speed & $20 \mathrm{~m} / \mathrm{s}$ \\
\hline Nodes position & Random \\
\hline
\end{tabular}

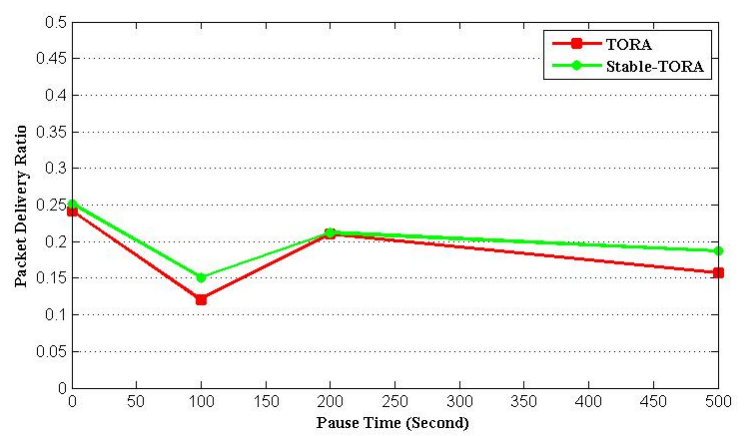

Fig. 5. Packet delivery ratio versus pause time.

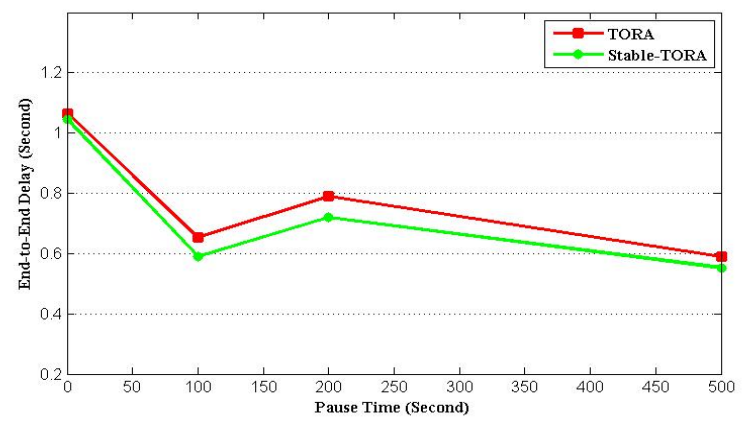

Fig. 6. End-to-end delay versus pause time

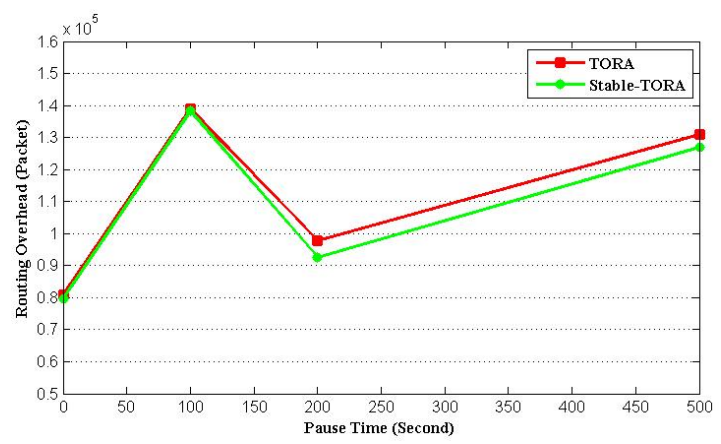

Fig. 7. Routing overhead versus pause time.

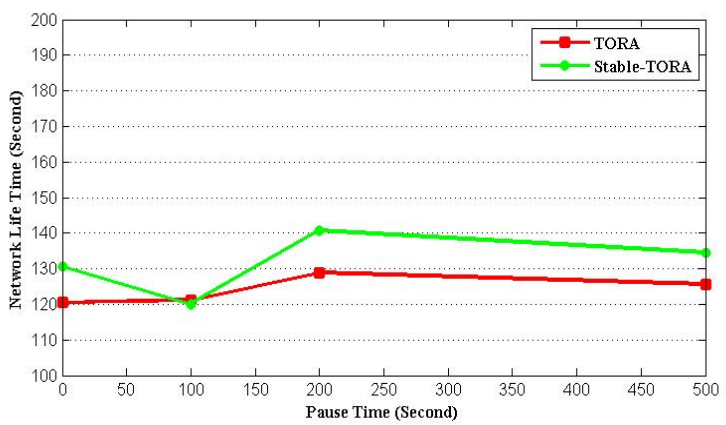

Fig. 8. Network life time versus pause time.

\section{CONCLUSION}

In this paper we introduced the Stable-TORA protocol, which is a routing protocol for mobile ad hoc networks. This protocol is based on the TORA routing protocol and has been provided in order to make this protocol more stable by discovery and use of high quality routes. Simulation results showed that the Stable-TORA protocol acts better in terms of packet delivery ratio, end to end delay, routing overhead, and network lifetime compared to TORA protocol, which is due to discovery and use of high quality routes. Research in this field should implement the same idea in other routing protocols in mobile ad hoc networks that do not consider stability in route discovery, and to provide new stable high quality protocols.

\section{REFERENCES}

[1] S. Sesay, Z. Yang. J. He, "A Survey on Mobile Ad Hoc Wireless Network", Information Technology Journal, Vol. 3, No. 2, pp. 168-175, 2004

[2] W. Kiess, M. Mauve, "A survey on real-world implementations of mobile ad-hoc networks", Ad Hoc Networks, Vol. 5, No. 3, pp. 324-339, 2007

[3] C. E. Perkins, P. Bhagwat, "Highly Dynamic Destination-Sequenced Distance Vector (DSDV) for Mobile Computers", SIGCOMM 1994 Conference on Communications Architectures, Protocols and Applications, pp. 234-244, 1994

[4] K. Akkaya, M. Younis, "A Survey on Routing Protocols for Wireless Sensor Networks", Ad Hoc Networks, Vol 40, No. 3, pp. 325-349, 2005

[5] N. Milanovic, M. Malek, A. Davidson, V. Milutinovic, "Routing and Security in Mobile Ad Hoc Networks", Computer, Vol. 37, No. 2, pp. $61-65,2004$ 
[6] M. Tarique, K. E. Tepe, S. Adibi, S. Erfani, "Survey of multipath routing protocols for mobile ad hoc networks", Journal of Network and Computer Applications, Vol 32, No. 6, pp. 143-152, 2009

[7] N.-C. Wang, C.-Y. Lee, "A multi-path QoS multicast routing protocol with slot assignment for mobile ad hoc networks", Information Sciences, Vol. 208, pp. 1-13, 2012

[8] S. Toy, S. Bandyopadhyay, T. Ueda, S. Tanaka, D. Saha, "An adaptive framework for multipath routing via maximally zone-disjoint shortest paths in ad hoc wireless networks with directional antenna", Global Telecommunications Conference, pp. 57-70, 2003

[9] C. Chen, M. Wu, S. Liu, S. Li, "A bidirectional symmetric routing protocol for MANETs with unidirectional links", IEEE International Conference of IEEE Region 10, pp. 1-4, 2013

[10] A. Kush, N.Garg, "Novel scheme for stable routing using battery status", $3^{\text {rd }}$ Intenational Conference on Computing for Sustainable Global Development, pp. 3309-3312, 2016

[11] F. Rump, S. A. Jopen, M. Frank, "Using Probabilistic Multipath Routing to Improve Route Stability in MANETs", IEEE 41st Conference on Local Computer Networks, pp. 192-195, 2016

[12] S. Sarkar, R. Datta, "An Adaptive Protocol for Stable and Energy-Aware Routing in MANETs", IETE Technical Review, Vol. 34, No.4, pp. 353365, 2016

[13] V. D. Park, M. S. Corson, "A Highly Adaptive Distributed Routing Algorithm for Mobile Wireless Networks", INFOCOM '97. Sixteenth Annual Joint Conference of the IEEE Computer and Communications Societies. Driving the Information Revolution, Vol. 3, pp. 1405-1413, 1997

[14] E. Perkins, Ad Hoc Networking, Addison-Weley, 2001

[15] B. S. Gouda, P. P. Panigrahi, R. K. Shial, "Efficient Optimal Route Stability Routing Protocol in MANET Using Gauss-Markov Mobility Model", IEEE $3^{\text {rd }}$ International Conference on Advances in Computing and Communications, pp. 445-448, 2013

[16] A. Zadin, T. Fevens, "Neighborhood-based interference minimization for stable position-based routing in mobile ad hoc networks", Future Generation Computer Systems, Vol. 64, pp. 88-97, 2016

[17] NS-2, The Network Simulator, http://nsnam.sourceforge.net/wiki/ index.php

[18] J. G. Sajjad, J. Shahram, V. K. Mina, "Energy Efficient Routing in Mobile Ad Hoc Networks by Using Honey Bee Mating Optimization", Journal of Advances in Computer Research, Vol. 3, No. 4, pp. 77-87, 2012

[19] M. K. Marina, S. R. Das, "On-demand Multipath Distance Vector Routing in Ad Hoc Networks", Ninth International Conference on Network Protocols, pp. 14-23, 2011

[20] S. Jamali, S. Jahanbakhsh, "BA-TORA: a Multipath Routing Protocol for MANETs by Inspiration from Bee and Ant Colonies", Przeglad Elektrotechniczny (Electrical Review), Vol. 2011, No. 7, pp. 183-187, 2011 